\title{
PERINATAL HEALTH AND TRANSLATIONAL MEDICINE
}

\author{
José Luiz Figueiredo ${ }^{1}$, Claudio Vinegoni' ${ }^{2}$, Luiz Carlos de Abreu ${ }^{3}$
}

Throughout fetal life, tissues and organs of the body are found at a critical period of development which coincides with the period of rapid cell division. When faced with lack of nutrients and hypoxia, the first fetal adaptation is a decrease in cell division rate. This cell division rate occurs both by direct effect of nitrogen starvation, and by hormonal and growth factors changes ${ }^{1}$. A reduction in the number of cells, the change in structure and functioning of organs, permanent change in DNA methylation, and in gene expression have also been considered to be molecular mechanisms responsible for fetal programming ${ }^{1}$.

On the other hand, epidemiological observations have led to the hypothesis that the risk of developing some noncommunicable chronic disease in adult is influenced not only by genetic sandadult life-style, but also by environmental factors acting early in life ${ }^{2}$. Environmental processes that influence the tendency for disease in adulthood appear to occur throughout the peri conceptual, fetal and infantile phases. Even short periods of malnutrition can permanently reduce the number of cells in certain organs during the fetal stage of development, causing changes in the distribution of cell types during metabolic activity, as well as throughout the organs' structure ${ }^{2}$. There is also growing evidence that maternal metabolism and intrauterine conditions may be responsible for the child 's development and growth programming ${ }^{1,2}$.

Intrauterine growth restriction (IUGR) occurs when the fetus does not reach the size determined by its genetic potential. This is a clinical problem that affects around $7-15 \%$ of gestations 3 and is associated with increased perinatal morbidity and mortality, as well as birth weight, which is understood to be an indicator of pregnancy conditions, and more specifically, intrauterine growth ${ }^{4-8}$. Low birth weight, disproportion in head circumference, and the length and weight at birth are markers of alack in nutrients at certain stages of pregnancy ${ }^{8,9}$. These changes, which reflect adaptations to support fetal development can permanently "program" the body's structure and function and thus be determinants for disease during adulthood; thus defining the fetal programming, as proposed by Barker ${ }^{1,2,9}$.

Children with low birth weight, regardless of their social background, have an increased risk of presenting cardiovascular disease and type 2 diabetes compared to their peers of higher weight ${ }^{10,11}$. Although factors related to adult weight and life-style may be additional confounding factors, the association of low birth weight with chronic diseases seems to persist after exclusion of such factors. There is a direct relationship between low birth weight and chronic disease, even after adjusting for relevant confounding variables, including alcohol consumption, levels of physical exercise and socioeconomic status ${ }^{10,11}$

Mortality in the perinatal period reflects generally the factors related to pregnancy and child birth, so, a significant number of deaths from preventable causes on the govern health services can be avoided. Increased attention to both mother and child is essential for the success of adequate growth and development of the individual ${ }^{12}$. Costa et al. ${ }^{8}$ observed a $25 \%$ incidence rate of underweight among infants born at term, and a large proportion of newborns' small-for-age ${ }^{12}$. The authors conducted an observational study, and reported that the incidence of insufficient birth weight (PNI) was one quarter of the total births ${ }^{12}$. In Brazil, $68.6 \%$ of newborn deaths under one year of age, occur in the neonatal period (up to 27 days of life), with the majority occurring on the first day of life. This rate seems to be related to low birth weight and adverse events during pregnancy ${ }^{12}$.

There is also evidence that the type of delivery is a corroborative factor for the implication of future outcomes, or a stimulator of epigenetic factors demonstrating the complexity of human growth and development. It is known that the most common issue associated with emergency or elective cesarean sections for a child's health, even for at term pregnancies, is respiratory disease, which increases perinatal mortality considerably ${ }^{11}$.

1 Division of Cardiovascular Medicine, Brigham and Women's Hospital, Harvard Medical School, Boston, MA, USA.

2 Center for Systems Biology, Massachusetts General Hospital, Harvard Medical School, Boston, MA, USA.

3 Department of Morphology and Physiology, School of Medicine of ABC, Santo Andre, SP, Brazil.

Correspondence: Luiz Carlos de Abreu. Department of Morphology and Physiology, School of Medicine of ABC, Santo André, SP, Brazil. E-mail: luiz.abreu@fmabc.br

Suggested citation: Figueiredo JL, Vinegoni C, de Abreu LC. Perinatal health and translational medicine. Journal of Human Growth and Development. 2013; 23(2): 125-127.

Manuscript submitted Aug 01 2013, accepted for publication Sep 192013. 
Epidemiological research have analyzed aspects such as mother's height, weight, ethnicity and parity variables, which are known to account for $20-35 \%$ of the variability of birth weight at term ${ }^{11}$. Research addressing newborns small for gestational age (SGA) and intrauterine growth restriction are well defined. However, those that relate to small for gestational age are quiescent ${ }^{8}$. Therefore, facing difficulties inherent toresearch with large population groups and miscegenation between peoples, is increasingly imperative incarrying out animal models to study the effects of low birth weight on off spring health, and its consequences on immediate and longterm growth and development.

Translational medicine, conceptually involves the set of knowledge designed in biomedical laboratories, tested on animals, which are then taken to the clinic in search for diagnostic and treatment improvements in tackling disease. Translational animal research is important for the development of criterias, and strict protocols, simulating with great accuracy, what may potentially happen to human health throughout life. As the vast majority of perinatal mortality is related to factors occurring during pregnancy, the creation of animal models is intrinsic for the replication and study of occurrences during this period.

Animal models of both growth restriction, and early cesarean section may serve to verify the effects, on an under weight model, of its under weight status, and of a cesarean inaccurately indicated, respectively. The use of arterial luminescent markers may identify changes in vessels during the first weeks of pregnancy, and provide information on what may occur in other organs after birth. In particular, recent advances in optical imaging of small animals combined with the use of fluorescence microscopy, the development of new molecular contrast enhancement, and new schemes available to compensate for motion artifacts, have opened possibilities for better observing biological and cellular physiology in vivo. The combination of imaging techniques, including radiological methods such as MRI, X-CT and CT PET, along with fluorescent microscopy may help in elucidating pathophysiological processes involved in the restriction of intrauterine growth, bringing new contributions to the current understanding.

Therefore, it is essential to incorporate basic research approaches that include: imaging methods, the involvement of multidisciplinary teams, a perspective on minimizing environmental and early interventionism impacts on child birth and development. Thus, the contribution by Costa et $\mathrm{al}^{8}$ is current and relevant for the field of public health. Investments should support the growth of knowledge in this area of expertise. Finally, translational medicine has shown to play an important role in address sing issues experienced faced by all of those continuously engaged in clinical and research practices. 


\section{REFERENCES:}

1. Chmurzynska A. Fetal programming: link between early nutrition, DNA methylation, and complex diseases. Nutr Rev. 2010 Feb;68(2):87-98. doi: 10.1111/j.17534887.2009.00265.x.

2. Gluckman PD, Hanson MA. Living with the past: evolution, development, and patterns of disease. Science. 2004;305:1733-1736.3.

3. Cetin I, Alvino G. Intrauterine growth restriction: implications for placental metabolism and transport. A review. Placenta 2009 Mar;30Suppl A:S77-82.

4. Donaldson PJ, Billy JOG. The impact of prenatal care on birth weight: evidence from an international data set. Med Care. 1984; 22: 177-88.

5. McIntire DD, Blomm SL, Casey BM, Leveno $\mathrm{KJ}$.Birth weight in relation to morbidity and mortality among newborn infants. N Engl J Med. 1999; 340(16):1234-8.

6. Puffer RR, Serrano CN. Caracteristicasdel peso ao nascer. Washington (DC) Organización Panamericana de la Salud; p.89-95 (OPASPublicación Científica, n504), 1988.

7. Rocha JA. Baixo peso, peso insuficiente e peso adequado ao nascer, em 5.940 nascidos vivos na cidade do Recife. J Pediatr (Rio J). 1991; 67(9/10):297-304.

8. Costa RS, et al. Incidence and characteristics of insufficient birth weight newborns to underweight from a cohort of neonates in a public hospital regional metropolitan area; J. Hum Growth and Dev. 2013; 23(2): 232-238

9. Baker, D.J.P. Maternal nutrition, fetal nutrition and diseade in later life. Nutrition.[S.I.], v.13, p. 807-813, 1997.

10. Bellingham-Young DA, Adamson-Macedo EN. The impact of birthweight on adult minor illness: a study on a sub-clinical population. J. Hum Growth and Dev 2013; 23(1):1-10.

11. Mattos SS, Chaves ME, Costa SM, Ishigami AC, Rêgo SB, SoutoMaior V, Severi R, de Lima FilhoJL. Which growth criteria better predict fetal programming? Arch Dis Child Fetal Neonatal Ed. 2013 Jan;98(1):F81-4. doi: 10.1136/ adc. 2010.207043.

12. Brasil. Ministério da Saúde. Secretaria de Atenção à Saúde. Departamento de Atenção Básica. Saúde da criança: crescimento e desenvolvimento / Ministério da Saúde. Secretaria de Atenção à Saúde. Departamento de Atenção Básica. - Brasília : Ministério da Saúde, 2012. 272 p.: il. - (Cadernos de Atenção Básica, no 33) ISBN 978-85-334-1970-4). P.23-26. 
